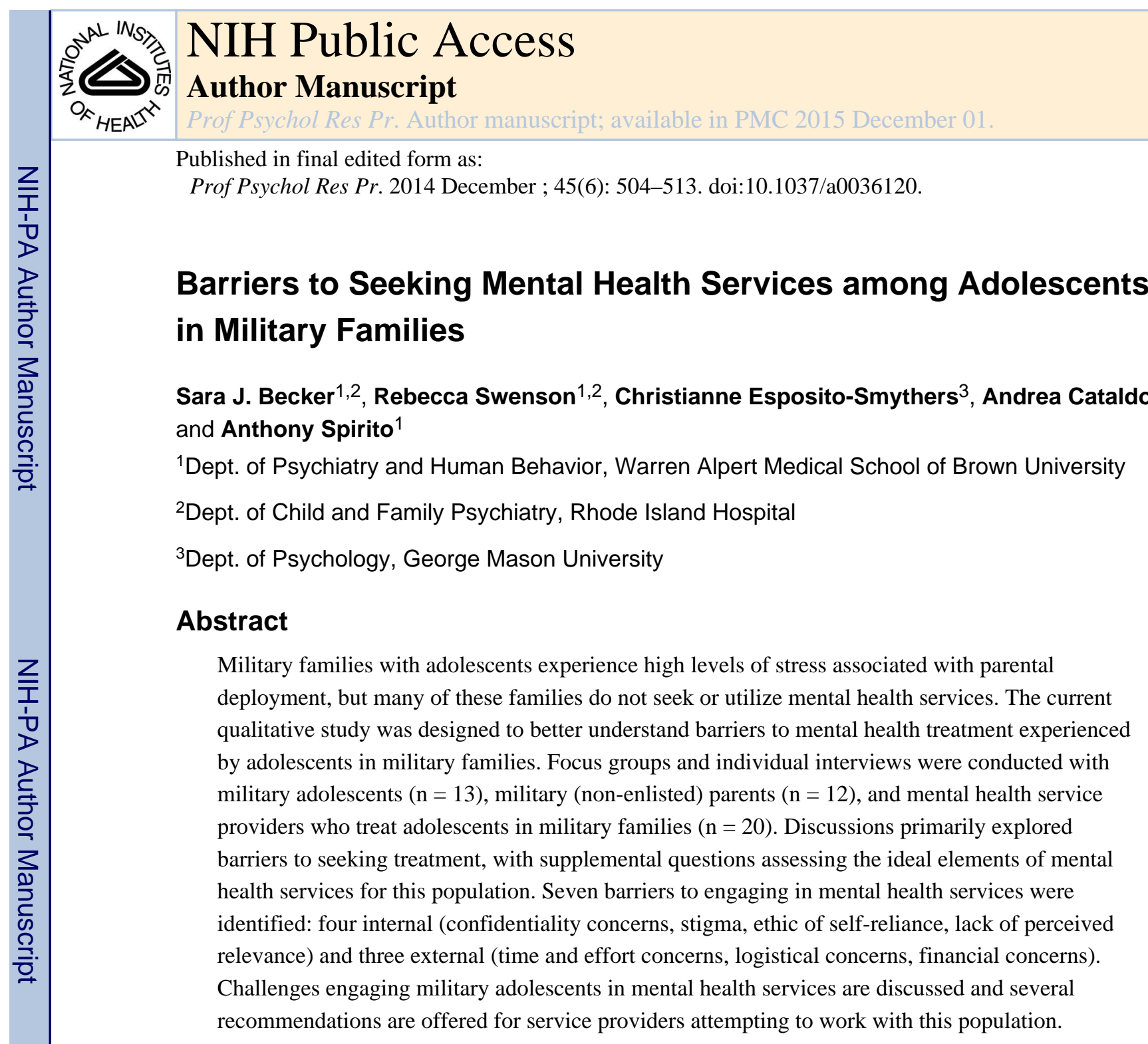

\title{
Keywords
}

military; adolescents; barriers; mental health

\begin{abstract}
Since the start of Operation Enduring Freedom in Afghanistan and Operation Iraqi Freedom, over 2 million children in the United States have been affected by the deployment of a parent (Chandra, Martin, Hawkins, \& Richardson, 2010). It has been estimated that over 30,000 of these youth have had to come to terms with parental death or injury (Lemmon \& Chartrand, 2009). Meanwhile, far greater numbers of youth have had to contend with stressors such as intermittent single parenting, financial strain, and frequent relocation.

Children in military families experience significant psychological and psychosocial stress associated with the deployment cycle. A recent study by Flake and colleagues (2009) found that children of service members are 2.5 times more likely to develop psychological
\end{abstract}

Correspondence regarding this article should be addressed to Sara Becker, Ph.D., Department of Psychiatry and Human Behavior, Warren Alpert Medical School of Brown University, One Hoppin Place, Suite 204, Providence, Rhode Island, 02903. Phone: (401) 793-8417. sara_becker@brown.edu. 
problems than American children in general. For youth, the deployment of a parent has been associated with depressive symptoms, externalizing behavior, academic problems, irritability, and impulsiveness (Esposito-Smythers et al., 2011; Jensen, Lewis, \& Xenakis, 1986; Medway, Davis, Cafferty, Chappell, \& O'Hearn, 1995). A study by Gorman and colleagues (2010) found that rates of behavioral and stress disorders among youth increased by about $18 \%$ during times of parental deployment, with a concurrent increase in mental health utilization of $11 \%$.

Parental deployment may be especially difficult during adolescence due to normative developmental changes including the onset of puberty. Adolescents in military families report a number of emotional and behavioral concerns, including loss of interest in activities, social withdrawal, changes in sleeping and eating, sadness, irritability, worry about their parent's safety, and disrespectful behavior (Huebner \& Mancini, 2005). Previous studies have found that the mental health symptoms experienced by teenagers in military families can lead to significant impairment in family, school, and social domains (e.g., Chandra et al., 2011; Chartrand, Frank, White, \& Shope, 2008; Houston et al., 2009; Lester et al., 2010; Mmari, Roche, Sudhinaraset, \& Blum, 2009). These mental health symptoms do not necessarily dissipate with the return of the military parent. Chandra and colleagues (2011) have found that the number of challenges experienced by military youth following the return of a deployed parent are influenced by multiple factors, including: the cumulative length of deployment, the non-deployed parent's mental state, and the age and gender of the youth (with girls and older teens experiencing more challenges during reintegration).

Despite these mental health challenges, rates of mental health utilization among military personnel are low. In a review of the literature, Ouimette et al. (2011) suggest that there are three main factors associated with low mental health utilization in military personnel: lack of confidence in and low perceived need for treatment, stigma-related fears, and beliefs in selfreliance. Hoge et al. (2004) surveyed combat veterans and found the most prevalent reasons for reluctance to seek mental health services included: cost (55\%), embarrassment (41\%), negative career implications (50\%), and being stigmatized by members of one's unit (59\%) as well as unit leadership (63\%). Data from the Mental Health Advisory Team's (U.S. Army, 2003) survey of deployed soldiers indicated that stigma was the most frequently identified barrier to mental health care, with particular concerns about how such care would be viewed by commanders and fellow unit members.

Although the literature has predominantly focused on service members, there is also concern that mental health problems are not adequately treated among military family members (American Psychological Association (APA) Presidential Task Force on Military Deployment Services for Youth Families and Service Members, 2007). For adolescents, in particular, it is well-documented that mental health services are underutilized (e.g., Gulliver, Griffiths, \& Christensen, 2010; Rickwood, Frank, \& Wilson, 2007). A recent systematic review by Gulliver and colleagues (2010) found that common barriers to seeking mental health services among adolescents in the general population included: stigma, concerns about confidentiality, lack of accessibility, and preference to rely on oneself. To date, no prior studies have explored specific barriers to service faced by adolescents in military 
families, even though these adolescents are at increased susceptibility to mental health problems.

The purpose of this study was to better understand barriers to mental health treatment experienced by adolescents in military families. To learn about potential barriers, we conducted qualitative research with military adolescents, military (non-enlisted) parents, and mental health service providers who treat adolescents in military families. Qualitative research can provide a vital source of information about patients' attitudes, perceptions, and experiences related to seeking mental health services as well as barriers that influence engagement. An understanding of factors that influence military adolescents' engagement in health services can potentially be used to improve outreach efforts and increase service utilization among this population.

\section{The Qualitative Research Approach}

\section{Target Population}

Three populations were targeted for the current study: 1) military adolescents with at least one active duty parent; 2) military (non-enlisted) parents of teenagers with an active duty spouse or partner; and 3) mental health service providers who have worked with adolescents from military families. Exclusion criteria were intentionally minimized to optimize the generalizability of the findings. Of note, we included any military adolescents or parents, regardless of whether or not the adolescents were experiencing emotional or behavioral problems that would require mental health treatment. This decision was made in order to avoid the exclusion of military family members who might have stigma-related concerns about acknowledging a mental health problem, as these concerns have been welldocumented in prior studies (Gulliver et al., 2010; Ouimette et al., 2011).

Specific inclusion criteria for the three target populations were: 1) military adolescents needed be 12 to 17 years old and have an active duty parent who had been deployed within the past 2 years; 2) military (non-enlisted) parents needed to be the biological parent or legal guardian of an adolescent who met the prior criteria; and 3) service providers needed to have offered mental health services to at least one adolescent from a military family within the past year. Participants also needed to possess sufficient language fluency and cognitive capacity to participate in a 1-hour interview or 2-hour focus group in English. Our target sample size was based upon guidelines published in a recent review paper by the National Centre for Research Methods (Baker \& Edwards, 2012): we aimed for a minimum of 12 participants per group and strived for saturation of key themes, defined as the point at which new data collection did not shed any further light on the research question.

\section{Recruitment}

Recruitment for this study occurred between August 2011 and April 2012. All three target populations (military adolescents, military parents, service providers) were recruited primarily through three outreach and support programs for military families in the northeast region: 1) Department of Defense Yellow Ribbon Reintegration ("Yellow Ribbon") Events; 2) National Guard Family Program; and 3) Operation Military Kids. The first program, Yellow Ribbon Events, consists of pre- and post-deployment events designed to prepare 
families for and provide support during the different phases of the deployment cycle. These full-day events are held 30 and 60 days prior to deployment as well as on a 45/90 or 30/60/90 day schedule upon return from deployments. Attendance at Yellow Ribbon Events is required by National Guard and Reserve Members, and the attendance of non-enlisted family members is encouraged. The second program, the National Guard Family Program, offers a wide range of optional services to military families, such as Family Readiness Groups (FRGs), educational events, support groups, social gatherings, financial assistance, and referrals for medical or mental health treatment. Finally, Operation Military Kids is a program funded by an Army Grant in collaboration with 4-H that provides a range of educational, training, and social events for military youth.

Study personnel developed relationships with staff in each of the above programs to identify specific events that would be appropriate to attend. At each event, study personnel offered an interactive workshop and/or set up an informational booth to provide education about common stressors related to parental deployment. All military families who attended the workshops and/or visited the information booths were given a brochure with a description of the qualitative research study. Interested families were invited to provide their contact information and subsequently called by the study research assistant for an eligibility screen. In addition, military families who contacted any of the above programs seeking mental health referrals were given information about the qualitative research study along with community referral options.

Mental health service providers were initially recruited through the same three programs. As study personnel developed relationships with program staff, providers who offered health services to military families were invited to participate in an interview. Additional providers were identified based on referrals from other providers and advertisements from local community agencies that were specifically designed for military families.

\section{Procedure}

The initial plan was to hold separate focus groups for the military parents and adolescents, consisting of 6-8 participants per group. However, consistent with the focus of this study, recruitment of military family members for events scheduled at specific times proved to be very difficult. Our team therefore offered military family members the choice to participate in either a small 60-90 minute focus group or a 30-60 minute individual interview.

Discussions were held at various times throughout the week to accommodate the family's schedules and preferences. Military parents and adolescents each received $\$ 50$ for their time and participation in the study.

The mental health service providers were all offered individual structured interviews. Because all of the providers were actively seeing patients, interviews were offered in-person or over the phone at various times. Service providers received $\$ 100$ for their time and participation.

Prior to commencement of each discussion, study personnel provided a verbal description of the study and obtained informed consent. In accordance with University Institutional Review Board procedures, written consent was provided by military parents and clinicians, 
while written assent was provided by military adolescents. The majority of the discussions were led by two Ph.D. level psychologists, each of whom has extensive training in clinical service delivery for adolescents and their families. A trained BA-level Research Assistant co-facilitated the focus groups and/or listened to audio recordings to take process notes.

\section{Discussion Guide}

Semi-structured focus group and interview protocols were used to guide discussions. To address the study's primary goal, focus group and interview questions were developed to generate feedback on barriers to engaging in mental health treatment among military families with adolescents. Questions pertaining to barriers were general and open-ended in order to elicit as many potential barriers to treatment as possible. In addition, a set of supplemental questions were designed to explore participants' views about the elements of an ideal mental health service. These supplemental questions were more targeted in nature. Four questions about ideal mental health services asked for participants' opinions on desirable service elements including: 1) delivery method (e.g., group versus individual); 2) frequency of sessions; 3 ) content of sessions (e.g., specific topics or skills); and 4) instructional methods.

\section{Qualitative Coding}

Discussions were digitally recorded and transcribed verbatim. Content related to barriers to seeking mental health services was qualitatively analyzed first. Based on a review of the extant literature, the study investigators developed a preliminary coding scheme consisting of two major themes: internal barriers (e.g., issues related to individual's beliefs, attitudes, and opinions) and external barriers (e.g., issues related to environmental or situational obstacles). These major themes were then divided into subordinate themes using principles of thematic analysis (Braun \& Clarke, 2006). In each discussion, emerging subordinate themes were checked iteratively against all other discussions and examined for trends, patterns, relationships, exceptions, and alternate explanations (Maxwell, 1992). The supplemental questions were then coded using structural (e.g., question-based) coding, since the same series of discrete questions were asked across all participants in the data set (Namey, Guest, Thairu, \& Johnson, 2007).

Thematic analysis proceeded in an iterative process involving two independent coders (the lead and second author). The first two discussions, which contained a total of 5 participants ( $11 \%$ of the total sample), were reviewed by the two coders separately. The coders then met to discuss emerging themes, determine a set of subordinate categories for coding, and develop definitions of the criteria for each category. Discussions with 10 individuals (22\% of the total sample) were then randomly selected using a random number generator for double coding. Transcripts were each coded independently and compared in order to calculate inter-rater reliability for each barrier. Because kappa values can excessively lower the estimate agreement in small samples (McHugh, 2012), both kappa values and percent agreement were calculated. The remaining discussions were coded by the lead author, and subsequently reviewed with the second author to reach consensus (100\% agreement). 
After comments were coded, frequency counts were obtained for each of the key themes. Individual participants were the unit of analysis, such that if one participant mentioned the same theme repeatedly, the theme was counted as having occurred once. This approach was based on the assumption that the number of individuals independently expressing the same idea was a better indicator of overall thematic importance than the absolute number of times the theme was articulated and coded (Namey et al., 2007). Consistent with guidelines by Meyer and Avery (2008), Excel was used as the qualitative analysis tool for the tracking, coding, and tabulating of verbatim comments.

\section{The Qualitative Research Sample}

\section{Military adolescents and parents}

A total of 13 military adolescents and 12 parents participated in the qualitative research. Of the 25 military family members, the majority were Caucasian $(n=20)$, with some representation of Hispanic $(n=4)$ and Other Race/Ethnicity $(n=1)$. About two-thirds of the 13 adolescents $(\mathrm{n}=8)$ were male and the average age was 13 years $(S D=1.4$, range from 12 to 17). All 12 of the military parents were female and identified as biological mothers. Average age of the parents was 42 years ( $S D=5.8$, range from 34 to 51). Among the parents, the most common levels of education were some college $(n=5)$ and college degree $(\mathrm{n}=10)$. When asked if they perceived their adolescent as experiencing behavioral or emotional problems, over half of the parents $(n=7)$ responded affirmatively.

Nine additional military parents gave their contact information at outreach events, but then did not enroll in the study; seven of these parents could not be contacted for an eligibility screen and two completed an eligibility screen but did not qualify. Reasons for exclusion included: 1) parent was not the legal guardian of an adolescent age 12 to 17 years; 2) parent was an active military member and did not have a non-enlisted spouse who was able to participate.

A total of 7 small focus groups (ranging in size from 2-4 individuals) and 6 structured interview discussions were conducted with the 25 military family members. One parent focus was conducted in a private room at a Yellow Ribbon Event, immediately following a brief workshop. All of the other discussions were conducted in a private room at an outpatient mental health clinic affiliated with an academic medical center.

\section{Mental health service providers}

A total of 20 mental health service providers participated, who were predominantly female $(n=17)$ and all Caucasian. Service providers were employed through military bases $(n=6)$, Veterans' Administration centers $(n=4)$, academic medical centers $(n=4)$, Universities ( $n$ $=2)$, or community agencies $(n=4)$. The majority $(n=15)$ of the service providers had at least MA-level education, and most identified themselves as primarily dedicated to $(\mathrm{n}=11)$ or moderately dedicated to $(n=4)$ serving military families.

All 20 service providers participated in individual interviews. Of the 20 interviews, 13 were conducted over the telephone, six were conducted at the outpatient mental health clinic described above, and one was conducted at a community location selected by the provider.

Prof Psychol Res Pr. Author manuscript; available in PMC 2015 December 01. 


\section{Themes Identified in the Qualitative Research}

In the following sections, results of the qualitative research are presented thematically with illustrative quotations. Within the text, frequency counts are presented using both the total number of occurrences across populations and the number of counts for each population. When frequency counts are presented, the letters $\mathrm{P}, \mathrm{A}$, and $\mathrm{C}$ are used to denote parents, adolescents, and clinicians, respectively.

\section{Barriers to Seeking Services}

Qualitative analysis resulted in the identification of seven barriers to seeking mental health services among military teenagers: four internal barriers and three external barriers. Table 1 presents each of the barriers, the kappa and inter-rater reliability (percent agreement) values, and their frequency counts across the full set of participants. Percent agreement was $90 \%$ or greater across all seven barriers, while kappa values ranged from .62 to 1.0, indicating substantial to perfect agreement (Cohen, 1960).

Internal barrier: Confidentiality concerns-The most frequently cited internal barrier to seeking mental health services across groups was confidentiality concerns $(\mathrm{n}=23 ; 7 \mathrm{P} ; 3$ A; 13 C). Among non-enlisted military parents, worries about confidentiality were typically related to the reputation of their active duty spouse or partner. Multiple parents $(n=4)$ stated that they would not be willing to seek services for their adolescent because they feared that their spouse's career would be negatively affected. In a focus group discussion with three mothers, one mother said "confidentiality is a concern because that might prevent your spouse from getting promoted," and the other two mothers adamantly agreed. In an individual interview, another mother noted that her husband was an officer and it could weaken his credibility if his direct reports learned his family had problems. Also related to the theme of confidentiality, several parents $(n=3)$ stated that they would want special assurance that their active duty spouse would not learn about their participation during a deployment. The primary concern of these parents was that their spouse would feel unduly burdened by problems while deployed. To illustrate, one mother said, "I don't want him thinking that we can't handle it, because then his mind isn't going to be on his mission and he is going to get himself killed."

Among adolescents, concerns about confidentiality tended to reflect general adolescent concerns and not necessarily specific concerns related to the military. Two teenagers stated that they did not want other teenagers or families in their community to find out that they were having a difficult time. One teenage boy said, "You might need to talk to someone, but there might be stuff you don't want everyone to know." Meanwhile, one other adolescent explicitly said that he would not be comfortable in any type of group programs due to worry that other participants would not respect his personal privacy.

Feedback from the clinicians was highly consistent with feedback from the military family members. Thirteen clinicians spontaneously identified confidentiality as one of the most significant barriers preventing military families from seeking mental health services. One clinician who practices on a military base said that she viewed educating families about confidentiality as a major component of her job. This clinician stated, "Families worry 
about treatment affecting the service member's career, even though it wouldn't. It's a matter of educating them that I won't report to any command." Several clinicians stated that they had adopted specific methods to protect military families' sense of privacy such as traveling to family homes, meeting families at an off-site location, or allowing families to come through a private entrance.

Internal barrier: Stigma-The next most frequently cited internal barrier to seeking services was stigma about therapy $(\mathrm{n}=22 ; 6 \mathrm{P}, 1 \mathrm{~A}, 15 \mathrm{C})$. While concerns about confidentiality were related to fear that others in the military community would find out about the family's therapy, concerns about stigma reflected negative views of therapy within the military family itself. A total of seven military family members $(6 \mathrm{P}, 1 \mathrm{~A})$ indicated that at least one of their family members had unflattering impressions of mental health treatment. Four military parents stated that their active duty spouse viewed therapy negatively or would judge them negatively for enrolling their child in treatment. As an example, one military parent said, "I know that my child's father avoids any type of therapy, so if he thought my son needed to go, he would be like, 'Hold on, time out, I don't do that." Meanwhile, another two parents stated that their adolescents had unflattering views of mental health treatment. In line with this view, one teen stated that she felt "embarrassed," and "weird" when her deployed parent returned home, because the parent knew that she had seen a therapist.

Seventy-five percent of the clinicians $(n=15)$ stated that they viewed stigma as a major barrier to seeking mental health services. Feedback from clinicians consistently indicated that this barrier is stronger among military families than non-military families. For instance, one service provider who works at a VA center stated, "In order to be a real soldier, admitting weakness is not acceptable, and mental health is considered a weakness. Even though the military has tried to break that belief down, I think its still completely prevalent." Similarly, a service provider who works on a military base said, "The attitudes about mental health among the military are probably 30-40 years back in terms of where they are publicwise." To address these negative views of therapy, several service providers described experimenting with different ways to promote their mental health services. For instance, multiple clinicians reported framing their services using universal terms such as resiliency training, stress management, or teen/parent support groups. Meanwhile, other clinicians reported combining mental health promotion with recreational activities targeted toward adolescents in order to increase the appeal of mental health services (e.g., having a movie or book discussion relevant to adolescents' mental health, having mental health professionals co-host a social outing for teens).

Internal barrier: Ethic of self-reliance-The third most commonly noted internal barrier to seeking services across groups was an ethic of self-reliance $(n=21 ; 3 \mathrm{P}, 4 \mathrm{~A}, 14$ C). Verbatim comments from the military family members (3 P, 4 A) suggested that this barrier was more pronounced among military families than among other family members with teenagers. Four military adolescents reported that they had a strong preference to solve problems without outside support. One teenage girl said, "I think that we military kids are more independent. If we have problems, we try and solve them ourselves or we go to the people we trust most, like our peers or our parents or an adult that we're very close with. I 
don't think we tend to gravitate to the therapy side of things, because we have to figure things out for ourselves all the time." Three parents similarly said that they would be reluctant to seek therapy for problems with their adolescent, because they preferred to either solve problems independently or turn to close family members or friends for help.

Consistent with this view, 14 clinicians stated that they had to acknowledge a strong sense of independence and self-sufficiency in order to work effectively with military families of adolescents. One clinician who worked at a VA stated, "There is a hurdle in dropping the guard and saying 'I'm struggling.' In the military the culture of self-sufficiency is so pervasive that you have to overcome that with the family members as well." Another clinician who worked at a community hospital stated, "I think there's a need to present as though everything is fine for military families during deployment as well as re-integration. This is the military way, no you don't have feelings or talk about them or acknowledge that they're hard." Four other clinicians made comments about how military families often preferred to "take care of their own," "to hunker down and become insular when issues arise," "to solve problems at the lowest level," and "to power through without help."

Internal barrier: Lack of interest or perceived relevance-The final internal barrier to seeking services was a lack of interest or perceived relevance $(n=18 ; 6$ P, 5 A, 7 C). In contrast to the other internal barriers, this barrier was cited more often by the military family members $(\mathrm{n}=11 ; 6 \mathrm{P}, 5 \mathrm{~A})$ than by the service providers $(\mathrm{n}=7)$. Two military teens specifically said that they would not be interested in any form of mental health services that required them to talk about their feelings. Another three teens made comments suggesting that therapy was not relevant to them because they didn't "need it," "need help," or "have problems." Along the same lines, six military parents made comments indicating that their teenagers would not be interested in or willing to engage in outside mental health services.

A total of seven service providers referenced this internal barrier to seeking services. Three service providers expressed concerns that parents might not view therapy as relevant due to difficulty differentiating between normative adolescent behavior and more significant mental health problems. Comments from these service providers indicated that this distinction was especially difficult during periods of parental deployment. One service provider at an academic medical center stated, "Families who are seeing their teens struggle, they think of it as a transient problem. They may say things like... 'Dad's away... this is normal. We don't need to do anything major like seek a mental health professional." Meanwhile, four other service providers referenced difficulties getting adolescents to view mental health services as relevant or appealing. These comments reflected a general lack of interest in therapy among teenagers and was not necessarily specific to the military. For instance, one service provider on a military base who hosts events to promote mental health said, "Teenagers just don't think what we are doing is cool."

External barrier: Time and effort concerns-In addition to the aforementioned internal barriers, participants acknowledged three external barriers to seeking mental health treatment. The most commonly mentioned external barrier was time and effort concerns $(\mathrm{n}=$ 31; $10 \mathrm{P}, 6 \mathrm{~A}, 15 \mathrm{C}$ ). For the military parents, time and energy concerns typically reflected the time constraints of single parenting during their spouse's deployment. As an example, 
one military mother said, "when your spouse is deployed, you are so busy being mom, dad, chef, washer, taxi driver, the whole 9 yards that there isn't time for anything else... it's a very difficult juggling act." Reflecting the same theme, another mother said, "Maybe the provider does not have weekend hours or evening hours so that's a big obstacle - not accessibility, but availability." Eight other parents made comments related to time constraints such as "there is never enough time," "it's a time restriction," and "we don't have time."

For the adolescents $(n=6)$, time and effort concerns reflected a more general barrier associated with the tendency of the teenagers to have exceptionally busy schedules. Comments from the teenagers referenced difficulties finding time for therapy sessions given their involvement in sports or extracurricular activities, or their parents' work schedules. Four adolescents specifically commented that they would only be available on weekends or during holiday vacations, one commented that she could never be available at the same time each week, and another said that he would only be available late in the evenings.

Fifteen service providers spontaneously acknowledged this barrier to treatment. Comments by the service providers indicated that time and effort concerns seemed to be a greater barrier for military families than for non-military families, especially in the midst of the deployment phase. As an example, one provider at an academic medical center said, "When there are deployments, the families are too busy and need to just keep their head above water. The first things to go are the appointments." One provider referenced the "compulsive busy-ness of the American family" and stated that this "busyness" was even more pronounced among military families. The remaining providers made similar comments about military families cancelling, rescheduling, or failing to comply with scheduled therapy sessions. To address this obstacle, the mental health providers reported making various accommodations, including: offering sessions and health promotions events at "off times" (e.g., nights and weekends); holding office hours during which patients could come for "drop in" sessions; and relaxing their usual attendance and cancellation policies.

External barrier: Logistical concerns-The next external barrier was logistical concerns $(\mathrm{n}=26 ; 8 \mathrm{P}, 4 \mathrm{~A}, 14 \mathrm{C})$ which included difficulties related to the logistics of attending an appointment such as transportation, commuting, parking, and childcare. Similar to time and effort concerns, this barrier tended to be most salient during periods of deployment when the non-deployed spouse was acting as a single parent. Reflecting the fact that this study occurred in a region without a centralized military base, a total of 8 military family members (7 P, $1 \mathrm{~A}$ ) commented that lack of access to transportation or the time required to commute to sessions was prohibitive for their family. Another four military families (3 P, $1 \mathrm{~A}$ ) mentioned issues with childcare as a barrier to seeking treatment. One military mother said, "The program would definitely have to provide for the whole family. If you didn't know for sure that your little ones are going to be taken care of, how can you go to a session or program and pay attention?"

The majority of the service providers $(\mathrm{n}=14)$ similarly acknowledged logistical issues as a major constraint to engaging military families in mental health services. Also supportive of the feedback from the military family members, the service providers independently 
mentioned transportation $(n=11)$ and childcare $(n=4)$ as two major logistical barriers. A number of the service providers reported making accommodations for military families, such as: offering home-based services; offering to meet in a centralized location near public transit; offering to provide transportation; and allowing parents of adolescents to bring infants and/or young children. Of these methods, home-based services was described as one of the most successful. For instance, one service provider affiliated with an academic medical center reported, "We've had military families say 'the only reason I'm doing this is because you can come when I'm available, I don't have to get in the car, and I don't have to find childcare to come to an appointment."

External barrier: Financial concerns-The last external barrier to seeking mental health services was financial concerns $(\mathrm{n}=17,4 \mathrm{P}, 1 \mathrm{~A}, 12 \mathrm{C})$. This concern reflected two related themes: 1) difficulties affording out of pocket fees ( $\mathrm{n}=10 ; 4 \mathrm{P}, 1 \mathrm{~A}, 5 \mathrm{C})$; and 2) limitations of some military insurance products in the coverage of mental and behavioral health services $(\mathrm{n}=8 ; 1 \mathrm{P}, 7 \mathrm{C})$. The first theme, challenges associated with out-of-pocket expenditures, was reported by four military parents and one military adolescent. One mother expressed her view that this concern was likely to be most common among families of junior rank, who had to live on a more restrictive budget. In harmony with this view, one teenage boy said, "You might want services to be free or really cheap so that my family and other families could go. I know some military families, they can't afford it." Five service providers similarly expressed concern that any out of pocket expenditures (e.g., session copayments, gas money) might present a barrier for military families. Two of these service providers specifically noted that there is an expectation within some military communities that services should be free, even among families with sufficient financial means.

The second theme, restrictions in insurance, was reported by only one parent who expressed uncertainty about her insurance plan's mental health coverage. By contrast, this theme was articulated by over a third of the mental health service providers $(n=7)$. Specific concerns pertained to: the level of reimbursement for the mental or behavioral health services provided by military insurance products; limitations in the quantity and quality of providers who accept military insurance products; and referral requirements prior to reimbursement. Two providers specifically noted that in order for their services to be covered, military families had to go through a centralized screening and referral process that might be viewed as prohibitive by some families. Reflecting a similar concern, two other providers reported treating military adolescents who had presented to an emergency department for routine mental health concerns due to difficulties finding outpatient services covered by their military insurance product.

\section{Elements of an Ideal Service Offering}

The final theme consisted of four targeted questions on attributes of ideal service offerings for military adolescents: format (e.g., group vs. individual); frequency; content; and instructional methods. Table 2 presents the frequency counts of the most common responses to the targeted questions across the full set of participants. 
Regarding the format of treatment, preferences were as follows: 28 participants $(7 \mathrm{P}, 11 \mathrm{~A}$, $10 \mathrm{C})$ said that they thought adolescents would benefit most from group-based treatment, 10 said a combination of group and individual ( $2 \mathrm{P}, 2 \mathrm{~A}, 6 \mathrm{C})$, and 7 said individual treatment (3 P, 4 C). Of note, the majority of participants in all three populations preferred group treatment, and none of the adolescents preferred individual treatment. About $25 \%$ of the total sample preferred a combination of group and individual treatment; the prevailing view of these participants was that groups would be appropriate for the majority of adolescents having emotional difficulties, whereas individual might be more appropriate for adolescents with a diagnosable or significantly impairing mental health condition. Of note, three of the four service providers who selected individual as the preferred treatment specifically said that they thought group-based services would be ideal for military teenagers, but that groupbased programs would not be realistic due to difficulties with attendance.

With regard to the frequency of services, the majority of participants across all three populations ( $\mathrm{n}=28 ; 7 \mathrm{P}, 8 \mathrm{~A}, 13 \mathrm{C}$ ) said that services should be offered on a periodic basis either "as needed" or at specific times during the deployment cycle (e.g., the weekend before deployment). Several other participants said that services should be offered weekly $(n=11$; $2 \mathrm{P}, 4 \mathrm{~A}, 5 \mathrm{C})$ or every other week $(\mathrm{n}=6$; $3 \mathrm{P}, 1 \mathrm{~A}, 2 \mathrm{C})$, although most participants indicated that the programs should have an open-door policy due to the challenges with regular attendance.

When asked an open-ended question about the type of content that would be most helpful, the four most common answers were related to: improving communication between the adolescent and other family members $(\mathrm{n}=22 ; 7 \mathrm{P}, 5 \mathrm{~A}, 10 \mathrm{C})$; basic education about the deployment cycle and its effects on the family $(\mathrm{n}=19 ; 6 \mathrm{P}, 3 \mathrm{~A}, 10 \mathrm{C})$; general stress management skills ( $\mathrm{n}=17 ; 3 \mathrm{P}, 2 \mathrm{~A}, 12 \mathrm{C})$; and education for the parent about adolescent behavior and related parenting skills $(\mathrm{n}=14 ; 5 \mathrm{P}, 1 \mathrm{~A}, 8 \mathrm{C})$. Of note, the most frequently suggested topics varied by population. Military family members most often suggested communication skills (7 P, $5 \mathrm{~A}$ ), whereas clinicians most often suggested stress management skills (12 C).

Finally, when asked an open-ended question about what instructional methods families with adolescents would find most beneficial, by far the most common response was methods that would facilitate social interactions $(n=24 ; 5$ A, 9 A, 10 C). About $75 \%$ of the teenagers $(n=$ 9) who participated in this study said that they would enjoy fun, social opportunities to connect with other military teenagers. Reflecting sensitivity to this view, ten service providers reported that they thought mental health services for military adolescents should include a fun, social component; of these service providers, six reported that they had offered recreational and/or social events that included a mental health component as part of their practice. These service providers all worked on the military base or in community agencies that were supported by grant funding to serve military youth. Examples of interactive, mental health promotion activities for military teenagers included wilderness adventure outings, photography clubs, arts and crafts projects, and seasonal outings (e.g., skiing in the winter, beach outings in the summer). One of the mental health providers who helped host these events, described them in the following manner, "Teens can come together because of the commonality of being military kids to support each other... and then as a 
clinical person, I can pull out individual people to offer support organically." Another provider described her approach to clinical work with military adolescents as "the vegetables in the meatloaf model," noting "we are talking about how to cope with deployment, but we are doing it in a fun, activity-based way." Of note, all six mental health providers who offered social or recreational activities for adolescents reported that they continued to have difficulties with attendance regardless of the event's stated objective.

Although not explored directly, a number of participants ( $\mathrm{n}=9$; $1 \mathrm{P}, 1 \mathrm{~A}, 7 \mathrm{C})$ spontaneously expressed interest in developing or using social media to enable military adolescents to network with one another. This idea was most often reported by clinicians, who shared the view that it would be therapeutic for adolescents to meet other military youth, either online or in person, regardless of the stated objective of the forum. One military youth similarly stated that he would benefit from an online social networking site, because, "You get to see all these other people that are dealing with the same thing you are and it's just a weight off your shoulders knowing you're not the only one in the entire world that's going through this. And you get this feeling inside of you, knowing that it's going to be okay."

\section{Summary of Qualitative Findings}

In order to elucidate barriers to mental health treatment experienced by adolescents in military families, we conducted qualitative research with military adolescents, military (nonenlisted) parents, and mental health service providers who treat military adolescents. Conducting interviews with three groups with potentially unique viewpoints represents a contribution of this study. Results identified four internal and three external barriers to engaging in treatment.

The four internal barriers - confidentiality, stigma, an ethic of self-reliance, and lack of perceived relevance - varied in the extent to which they reflected issues specific to military families. Confidentiality and stigma concerns for military adolescents were consistent with general adolescent concerns about personal privacy that have been documented in the literature (e.g., citation). By contrast, for military parents, confidentiality and stigma concerns were related to concerns that having their teen receive mental health counseling might be viewed negatively by their spouse or by the military community. Clinicians also viewed these concerns as unique to military families and reported addressing these concerns through education and accommodations such as off-site or home-based sessions. For some military parents, the confidentiality and stigma concerns were so significant that they feared that if the deployed spouse found out the teen was receiving services, the deployed spouse would experience stress and be indirectly placed at greater risk of injury and death. The countervailing view that a deployed parent might feel better knowing that their teen was receiving mental health services was never mentioned. This feedback suggests room for improvement in the military's attempts to destigmatize therapy and to educate personnel that their family's receipt of mental health services will not affect their careers. Perhaps a different way to affect the negative attitude toward receipt of mental health services by families would be to restate the message as follows: Knowing that family members are 
receiving services can give our service members peace of mind, and this peace of mind can improve the performance of our troops.

Another significant barrier that was unique to military adolescents was an ethic of selfreliance. While self-reliance has been identified as a barrier to mental health care in the general adolescent population (Gulliver et al., 2010), multiple participants expressed the view that military youth are more likely to be self-reliant than non-military youth. This ethic of self-reliance may contribute to the final internal barrier - lack of perceived interest or relevance of mental health services. A number of military parents and adolescents felt that it was "normal" to be stressed when a parent was deployed; consequently, it seemed as if many military family members viewed mental health symptoms during the deployment cycle as temporary and as not requiring professional care. Professionals, on the other hand, were concerned that because of this attitude, parents may not recognize when an adolescent is in genuine need of services.

Parents, adolescents, and providers uniformly stated that external barriers, such as time and effort concerns, logistical concerns, and financial concerns, directly affected the use of mental health services. Time and effort concerns and logistical concerns were more frequently reported than any of the internal barriers. While these issues may be shared by nonmilitary families, many of these issues are likely to be more prominent in military families due to the burden of single parenting during periods of deployment. In addition, there were some specific financial concerns related to the levels of mental health coverage under particular military insurance products.

The pervasiveness of these barriers to seeking service was reflected in the responses to our questions about the ideal elements of mental health services. Feedback from the military families emphasized a desire for regular opportunities to connect with other military families both in-person and using technology. Consistent with this view, military family members were predominantly interested in services that were offered in group format. Meanwhile, feedback from clinicians emphasized persistent difficulties with attendance, even when events had a prominent recreational or social element. As a result, half of the 20 clinicians (n $=10$ ) thought that mental health services should be offered in group format, whereas the other half thought that individual treatment or a combination of individual and group treatment would be more beneficial. Of note, multiple clinicians specifically stated that they did not view group treatment as feasible due to the difficulties getting families to attend mental health services with any degree of regularity. Finally, responses to the question about the ideal content of mental health services emphasized a combination of general skills (e.g., parenting, communication, stress management) with specific psychoeducation for military families (e.g., deployment cycle, effects of deployment on families). This feedback was consistent with published guidelines for military youth (Esposito-Smythers et al., 2011) and suggests that adolescents in military families may not require new intervention models as much as tailoring of existing models to provide relevant examples and education.

Results of this qualitative study are affected by several limitations. First, it is important to acknowledge that this study used purposive sampling to recruit military adolescents and parents. All of the military adolescents and parents who participated first had to learn about 
the study via our specified recruitment methods, and then had to be able and willing to participate in an interview or focus group. It is therefore possible that our recruitment methods did not reach other military families who might have been even more significantly affected by barriers to engaging in services. Second, we had planned to use focus groups but had difficulty gathering participants together and therefore conducted most interviews individually. It is possible that some different and/or additional comments and ideas may have been generated in focus groups due to social facilitation that were not elicited in the individual interviews. Third, the military adolescents did not have to have a mental or behavioral health problem to participate in the study, and about half of the parents did not identify their adolescent as having a mental or behavioral health concern. A different pattern of results might have been reported by adolescents and parents who were significantly impaired by a mental health problem. Finally, participants in the current study were recruited in a region without a central military base. Barriers to participation might differ in other regions in which military communities are more centralized.

When considering the results of this study, it is important to note that there was a great deal of consistency in the specific themes mentioned by military family members and mental health service providers. However, there was some variability in the frequency with which themes were mentioned. For instance, several barriers were mentioned by a greater proportion of clinicians than military family members (e.g., stigma, ethic of self-reliance, financial concerns), whereas one barrier (e.g., lack of perceived relevance) was mentioned by a greater proportion of military family members. One interesting direction for future research would be to evaluate whether the impressions of clinicians or military family members are more likely to predict actual treatment seeking behavior.

\section{Conclusion}

In conclusion, the current qualitative study identified four internal barriers (e.g., stigma, confidentiality concerns, ethic of self-reliance, and lack of relevance) and three external barriers (e.g., time and effort concerns, logistical concerns, and financial concerns) to engaging in mental health services among military families with adolescents. The internal barriers, in particular, appeared to reflect concerns unique to military families and were highly consistent with barriers identified in prior literature on military personnel. The external barriers were predominantly reflective of difficulties accessing mental health treatment when the service member was deployed, similar to the types of obstacles encountered by single parent families. In isolation, none of the seven barriers to treatment seemed to be insurmountable and, indeed, many of the service providers could provide concrete examples of ways they had attempted to address these barriers. However, when combined, these seven barriers raise unique challenges to engaging military families with teenagers in treatment, as evidenced by the persistent attendance challenges faced by providers who offered social, supportive, or recreational events. Taken together, our findings suggest that service providers attempting to work with military families need to adopt a multi-faceted approach that: considers issues of framing (e.g., describing events using universal language), provides education about the benefits of mental health treatment for the entire family (including the service member), and offers significant flexibility with regard to the timing, cost and location of services. Home-based services and technology-assisted 
support groups represent examples of models that might serve to address some of the barriers faced by this population.

\section{Acknowledgments}

The initial project and data collection were supported by Grant 1R34 MH082164-01A2 from the National Institute of Mental Health. A portion of the time spent on the analysis and writing of this manuscript was funded by Grant 1K23DA031743-01 from the National Institute on Drug Abuse to Sara Becker.

\section{References}

American Psychological Association (APA) Presidential Task Force on Military Deployment Services for Youth Families and Service Members. The Psychological Needs of US Military Service Members and Their Families: A Preliminary Report. 2007. Retrieved from http://www.apa.org/ releases/MilitaryDeploymentTaskForceReport.pdf

Baker, SE.; Edwards, R. How many qualitative interviews is enough?: Expert voices and early career reflections on sampling and cases in qualitative research. Southampton: National Centre for Research Methods; 2012.

Braun V, Clarke V. Using thematic analysis in psychology. Qualitative Research in Psychology. 2006; 3:77-101.10.1191/1478088706qp063oa

Chandra, A.; Lara-Cinisomo, S.; Jaycox, LH.; Tanielian, T.; Han, B.; Burns, RM.; Ruder, T. Views from the homefront: The experiences of youth and spouses from military families. Santa Monica, CA: RAND Corporation; 2011.

Chandra A, Martin LT, Hawkins SA, Richardson A. The impact of parental deployment on child social and emotional functioning: Perspectives of school staff. Journal of Adolescent Health. 2010; 46:218-223.10.1016/j.jadohealth.2009.10.009 [PubMed: 20159497]

Cohen J. A coefficient of agreement for nominal scales. Educational and Psychological Measurement. 1960; 20:37-46.

Chartrand MM, Frank DA, White LF, Shope TR. Effects of parents' wartime deployment on the behavior of young children in military families. Archives of Pediatrics \& Adolescent Medicine. 2008; 162:1009-1014.10.1001/archpedi.162.11.1009 [PubMed: 18981347]

Esposito-Smythers C, Wolff J, Lemmon KM, Bodzy M, Swenson RR, Spirito A. Military youth and the deployment cycle: Emotional health consequences and recommendations for intervention. Journal of Family Psychology. 2011; 25:497-507. [PubMed: 21707172]

Flake EM, Davis BE, Johnson PL, Middleton LS. The psychosocial effects of deployment on military children. Journal of Developmental and Behavioral Pediatrics. 2009; 30:271-278.10.1097/DBP. 0b013e3181aac6e4 [PubMed: 19606059]

Gorman GH, Eide M, Hisle-Gorman E. Wartime military deployment and increased pediatric mental and behavioral health complaints. Pediatrics. 2010; 126:1058-1066.10.1542/peds.2009-2856 [PubMed: 21059715]

Gulliver A, Griffiths KM, Christensen H. Perceived barriers and facilitators to mental health helpseeking in young people: A systematic review. BMC Psychiatry. 2010; 1010.1186/1471-244x-10-113

Hoge CW, Castro CA, Messer SC, McGurk D, Cotting DI, Koffman RL. Combat Duty in Iraq and Afghanistan, Mental Health Problems, and Barriers to Care. The New England Journal of Medicine. 2004; 351:13-22.10.1056/NEJMoa040603 [PubMed: 15229303]

Houston JB, Pfefferbaum B, Sherman MD, Melson AG, Jeon-Slaughter H, Brand MW, Jarman Y. Children of deployed National Guard troops: Perceptions of parental deployment to Operation Iraqi Freedom. Psychiatric Annals. 2009; 39:805-811.10.3928/00485713-20090728-03

Huebner, AJ.; Mancini, JA. Adjustments among adolescents in military families when a parent is deployed. Final Report to the Military Family Research Institute and Department of Defense Quality of Life Office. 2005. Retrieved from http://www.cfs.purdue.edu/mfri/pages/research/ Adjustments_in_adolescents.pdf 
Jensen PS, Lewis RL, Xenakis SN. The military family in review: Context, risk, and prevention. Journal of the American Academy of Child Psychiatry. 1986; 25:225-234.10.1016/ s0002-7138(09)60230-2 [PubMed: 3700910]

Kelley ML, Hock E, Smith KM, Jarvis MS, Bonney JF, Gaffney MA. Internalizing and externalizing behavior of children with enlisted Navy mothers experiencing military-induced separation. Journal of the American Academy of Child \& Adolescent Psychiatry. 2001; 40:464471.10.1097/00004583-200104000-00016 [PubMed: 11314573]

Lemmon KL, Chartrand MM. Caring for America's children: Military youth in time of war. Pediatrics Review. 2009; 30:42-48.

Lester P, Peterson K, Reeves J, Knauss L, Glover D, Mogil C, Beardslee W. The long war and parental combat deployment: Effects on military children and at-home spouses. Journal of the American Academy of Child \& Adolescent Psychiatry. 2010; 49:310-320. [PubMed: 20410724]

Maxwell JA. Understanding and validity in qualitative research. Harvard Educational Review. 1992; 62:279-300.

McHugh ML. Interrater reliability: The kappa statistic. Biochemia Medicma. 2012; 22:276-282. http:// dx.doi.org/10.11613/BM.2012.031.

Medway FJ, Davis KE, Cafferty TP, Chappell KD, O'Hearn RE. Family disruption and adult attachment correlates of spouse and child reactions to separation and reunion due to Operation Desert Storm. Journal of Social and Clinical Psychology. 1995; 14:97-118.10.1521/jscp. 1995.14.2.97

Meyer DZ, Avery LM. Excel as a qualitative data analysis tool. Field methods. 2009; 21:91112.10.1177/1525822X08323985

Mmari K, Roche KM, Sudhinaraset M, Blum R. When a parent goes off to war: Exploring issues faced by adolescents and their families. Youth \& Society. 2009; 40:455-475.

Namey, E.; Guest, G.; Thairu, L.; Johnson, L. Data reduction techniques for large qualitative data sets. In: Guest, G.; MacQueen, K., editors. Handbook for Team-based Qualitative Research. Lanham, MD: AltaMira Press; 2007. p. 137-162.

Ouimette P, Vogt D, Wade M, Tirone V, Greenbaum MA, Kimerling R, Rosen CS. Perceived barriers to care among veterans health administration patients with posttraumatic stress disorder. Psychological Services. 2011; 8:212-223.

Rickwood D, Frank D, Wilson C. When and how do young people seek professional help for mental health problems? Medical Journal of Austrailia. 2007; 187:35-39.

U.S. Army. Operation Iraqi Freedom (OIF) Mental Health Advisory Team (MHAT) Report. 2003. Retrieved from http://www.pbs.org/wgbh/pages/frontline/shows/heart/readings/mhat.pdf

Prof Psychol Res Pr. Author manuscript; available in PMC 2015 December 01. 


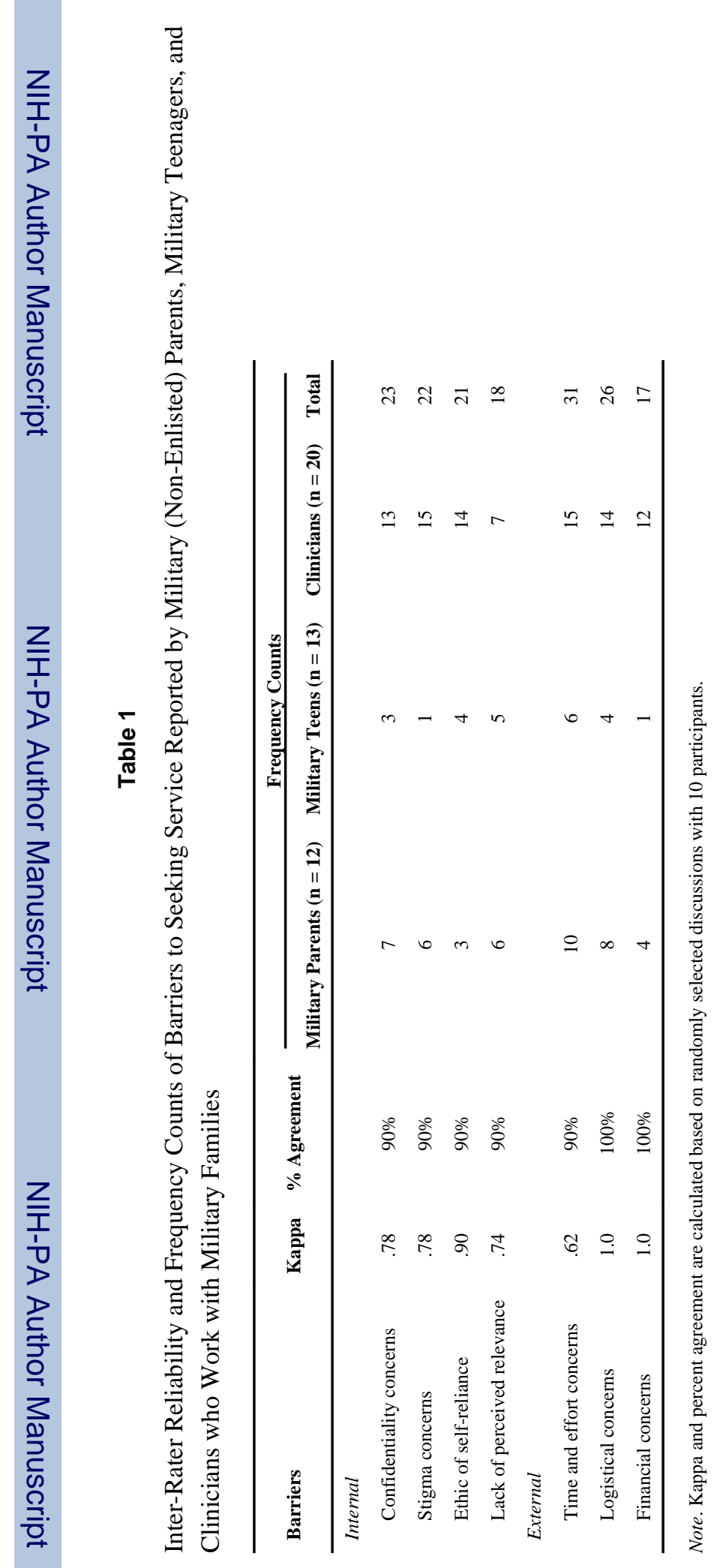

Prof Psychol Res Pr. Author manuscript; available in PMC 2015 December 01. 


\section{Table 2}

Frequency Counts of Responses to Targeted Questions by Military (Non-Enlisted) Parents, Military Teenagers, and Clinicians who Work with Military Families

\begin{tabular}{|c|c|c|c|c|}
\hline \multirow{2}{*}{ Responses } & \multicolumn{4}{|c|}{ Frequency Counts } \\
\hline & Military Parents ( $n=12)$ & Military Teens $(n=13)$ & Clinicians $(\mathrm{n}=\mathbf{2 0})$ & Total \\
\hline \multicolumn{5}{|l|}{ Preferred Modality } \\
\hline Group & 7 & 11 & 10 & 28 \\
\hline Individual & 3 & 0 & 7 & 10 \\
\hline Combination & 2 & 2 & 6 & 10 \\
\hline \multicolumn{5}{|l|}{ Preferred Frequency } \\
\hline Weekly & 2 & 4 & 5 & 11 \\
\hline Biweekly or Monthly & 3 & 1 & 2 & 6 \\
\hline Periodic or As Needed & 6 & 8 & 8 & 22 \\
\hline \multicolumn{5}{|l|}{ Preferred Content } \\
\hline Communication & 7 & 5 & 10 & 22 \\
\hline Psychoeducation & 6 & 3 & 10 & 19 \\
\hline Stress Management & 3 & 2 & 12 & 17 \\
\hline Parenting & 5 & 1 & 8 & 14 \\
\hline \multicolumn{5}{|l|}{ Preferred Delivery Elements } \\
\hline Social or "fun" & 5 & 9 & 10 & 24 \\
\hline Uses technology & 1 & 1 & 7 & 9 \\
\hline
\end{tabular}

Prof Psychol Res Pr. Author manuscript; available in PMC 2015 December 01. 